\title{
CLASIFICACIÓN DE LAS COMUNIDADES VEGETALES EN LA REGIÓN ÁRIDA DEL ESTADO DE CHIHUAHUA, MEXICO
}

\section{CLASSIFICATION OF PLANT COMMUNITIES OF THE ARID REGION OF CHIHUAHUA, MEXICO}

\author{
Manuel Sosa ${ }^{1}$, José Luis Galarza ${ }^{2}$, Toutcha Lebgue ${ }^{3}$, Ricardo Soto ${ }^{4}$ y Soraya Puga ${ }^{5}$
}

\section{Resumen}

Las comunidades vegetales del desierto, se caracterizan por su fragilidad y condiciones muy específicas debido a los factores medioambientales. El ecosistema desierto, tiene gran importancia no sólo por la gran superficie que ocupa sino por sus características específicas y diversidad de especies que presenta, muchas de ellas en riesgo. El objetivo del estudio fue definir las clases, asociaciones vegetales y la superficie que ocupan en la zona de estudio utilizando valores de cobertura vegetal y presencia de especies. Se muestrearon 110 sitios utilizando la metodología del Gap Análisis. Se aplicó un análisis multivariado de conglomerados y se usó la técnica de varianza mínima de Ward. El resultado reflejó similaridad entre lo sitios observados de $\mathrm{r}^{2}=80.93 \%$, por lo que se obtuvo un buen grado de confiabilidad de las agrupaciones; se identificaron 3 grupos con 2 conglomerados cada uno. El grupo 1 con 23 sitios y 9 especies dominantes, el grupo 2 con 28 sitios y 2 especies dominantes y el grupo 3 con 58 sitios y 7 especies dominantes. Las especies que están presentes en mas del 50\% del área de estudio fueron: Larrea tridentata y Prosopis glandulosa.

Palabras Claves: Asociaciones vegetales, vegetación desértica, conglomerados, biodiversidad

\begin{abstract}
Desert plant communities are characterized by specific fragile conditions due to environmental factors. The Desert Ecosystem plays a very important role, not only for the wide area occupied but also for its specific characters and species diversity, some of which are considered endangered. The objective of this study was to define classes, vegetation associations and area occupied within the study zone using plant cover values and presence of plant species. Data were obtained from 110 sampling sites, through the GAP Analysis methodology. Cluster analysis method to group homogeneous classes with plant cover and species structure applying the Ward minimum variance technique was used. Results depicted similarity among observed sites $(\mathrm{r} 2=80,93)$ which means good confidence in the associations; three groups were identified with 2 clusters each. Group 1 with 23 sites and 9 dominant species; group 2 with 28 sites and 2 dominant species; and group 3 with 58 sites and 7 dominant species. Plant species present in the area with more than $50 \%$ cover are: Larrea tridentata and Prosopis glandulosa.
\end{abstract}

Key words: Vegetation Associations, desert vegetation, clusters, biodiversity

\section{Introducción}

La distribución de las comunidades vegetales dependen de las relaciones existentes entre características fisiográficas y climáticas de la región y las características intrínsecas de las especies vegetales. La región árida y semiárida cubre un área de 103 millones de ha, es decir, el $52 \%$ de la superficie continental de México, constituyéndola lomeríos y grandes planicies situadas desde el nivel del mar hasta alrededor de 2400 msnm (Solís, 1992).

El análisis y clasificación de la vegetación de la zona árida del estado de Chihuahua, comprendida dentro del ecosistema Desierto Chihuahuense, toma como fundamento la variable cobertura vegetal de las especies presentes, dentro de la fisionomía de las asociaciones vegetales. La cobertura vegetal, es una variable biofísica que está integrada a los procesos biogeoquímicos, hidrológicos y en la interacción de la superficie terrestre con la atmósfera (Cihlar et al., 2000).

La comunidad vegetal presenta un conjunto de atributos cuyo significado demuestra el nivel de integración de una comunidad, por lo que gran parte de las investigaciones en ecología de comunidades han estado dirigidas a medir los niveles de asociaciones entre las especies (Krebs, 1985).

El Desierto Chihuahuense, en su porción dentro del estado de Chihuahua es una de las áreas menos estudiadas en cuanto a biodiversidad ecológica, siendo los bosques y áreas de pastizal donde se cuenta con mayor investigación (Estrada, 1995).

La región desértica presenta condiciones restringidas de humedad disponible en el suelo y se encuentra limitada a una estación muy corta de 
precipitación al año, lo que la caracteriza como zona árida; esto permite que sus organismos vivos como la vegetación y los animales tengan que evolucionar para adaptarse a estas características extremas de su medio (Soto et al., 2000)

El paisaje consiste en superficies de terreno en donde las comunidades vegetales son de porte arbustivo y se caracterizan como matorrales xerófitos (Rzedowski, 1978). Las plantas están muy dispersas, con mucho suelo desnudo entre ellas y las precipitaciones en estas regiones desérticas suelen ser de $250 \mathrm{~mm}$ o menos, aunque algunas veces con mayor cantidad pero distribuidas irregularmente (Solís, 1992).

Las comunidades vegetales y su ambiente forman un sistema funcional que define un ecosistema (Whittaker, 1972). Siendo la comunidad un nivel de formando parte de un ensamble de poblaciones que viven juntas en una sola área (Krebs, 1998).

El objetivo de este estudio fue analizar, definir y cuantificar las clases de vegetación de la región árida del estado de Chihuahua, México.

\section{Materiales y Métodos}

Ubicación geográfica. El área se localiza en la porción noreste del estado de Chihuahua y en la parte media del Desierto Chihuahuense (Figura 1), geográficamente en las coordenadas $104^{\circ} 40^{\prime} 00^{\prime}$ ' longitud oeste y $29^{\circ} 29^{\prime} 28^{\prime}$ ' Latitud Norte, extremo Noroeste y en las coordenadas $103^{\circ} 39^{\prime} 58^{\prime \prime}$ Lo y $28^{\circ}$ 29' 38' extremo Sureste (INEGI, 1976, 1977 y 1978).

Fisiografía. El área de estudio presenta características fisiográficas muy heterogéneas, con rangos que oscilan desde los $680 \mathrm{msnm}$, en la zona de influencia

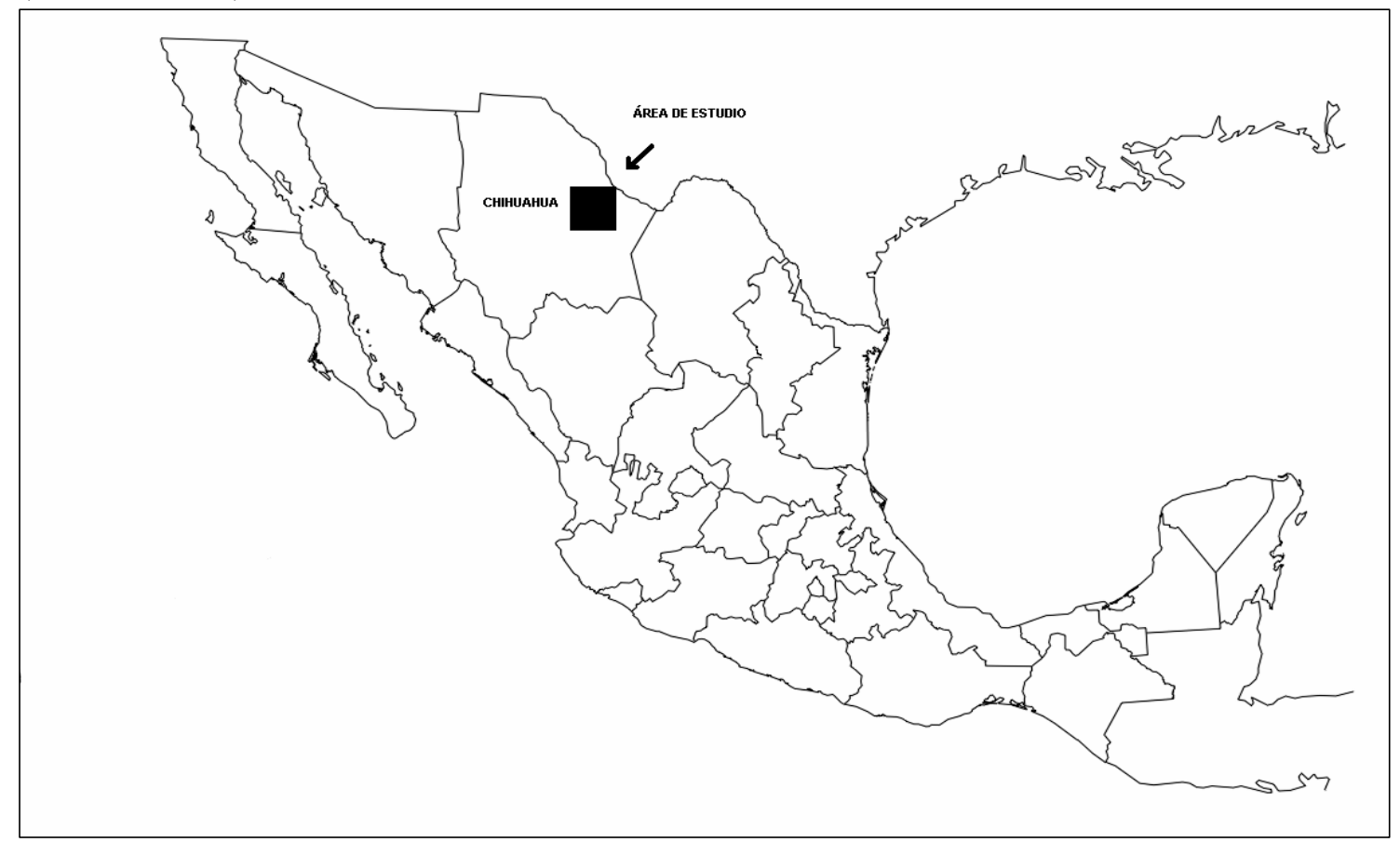

Figura 1.- Localización del área de estudio.

organización derivado de los ecosistemas, que consiste en un conjunto de organismos vivos de diversas especies que comparten un mismo ambiente con una cierta particularidad distintiva (Ondarza, 1993).

Para su mejor entendimiento y manejo, es necesario fragmentarlo, resultando mas conveniente estudiar unidades o grupos pequeños de especies, manejando separadamente cada variable (Fierro et al., 1980) sin olvidar que todas las observaciones y mediciones realizadas deben encuadrarse en un marco ecológico.

Las comunidades vegetales dividen sus características en cualitativas y cuantitativas, y está constituida por organismos y poblaciones de especies, al Río Bravo, hasta los 2400 msnm de la parte más alta de Sierra Rica. En el centro de la región de estudio se da un gradiente que es el de mayor presencia en la zona con un rango entre los 1360 a 1 440 msnm.

Edafología. Los suelos presentan condiciones muy diversas. Según la clasificación de INEGI, los suelos son del tipo Regosol, con características de formación in situ y coluvial, derivado principalmente de las rocas calizas, muy gravosos y de origen ígneo con una profundidad muy somera (de 0 a $25 \mathrm{~cm}$ ). Los suelos del tipo Xerosol, generalmente de origen aluvial y profundos (> a $50 \mathrm{~cm}$ ), los colores son generalmente pardos oliva a pardos amarillento, pueden presentar texturas franco - arcillosas o arcillosas. Los suelos 
Litosoles, generalmente están asociados a las formaciones rocosas, son depósitos de rocas calizas, rocas sedimentarias e ígneas extrusivas, no presentan ningún perfil de suelo y su estructura es de consistencia sólida y frágil en aquellas montañas poco consolidadas, presentan escurrimientos muy acelerados y pendientes mayores a $26 \%$.

Vegetación. La vegetación del área se caracterizan por ser de tipo xerófita compuesta por matorrales micrófilos y rosetófilos. Los matorrales micrófilos se localizan principalmente en las conformaciones de valles y suelos con depósitos aluviales, y en las formaciones de lomeríos suaves, ocupan la mayor parte del área. Dentro de los estratos de matorrales inermes (1 a $2 \mathrm{~m}$ de altura), destacan especies como Artemisia filifolia (Artemisa), Flourensia cernua (hojasén), Parthenium incanum (mariola) y Larrea tridentata (gobernadora). El matorral subinerme o mediano espinoso, está representado principalmente por arbustos de complexión mediana (1.5 a $2 \mathrm{~m}$ de altura) destacando: largoncillo (Acacia constricta), mezquite (Prosopis glandulosa o juliflora), gatuño (Mimosa biuncifera), junco (Koeberlinia spinosa), grangel (Celtis pallida) y ocotillo (Fouquieria splendes).

Hidrología. El área de estudio se encuentra en la Región Administrativa No. VI de la frontera norte del Río Bravo, dividida por la cuenca del Conchos y Alto Bravo (CNA y SEMARNAP, 2000). El principal cause dentro del área es el río Bravo (Río Grande en USA) con corrientes permanentes y una longitud de casi 80 km dentro del área.

Clima. González (2001), reporta un clima de tipo BWhw esto es clima árido o desértico (BW), con temperaturas medias anuales de $18^{\circ} \mathrm{C}(\mathrm{h})$ y lluvias de verano (w) la precipitación media anual es de 286.1 mm y las temperaturas medias anuales máximas y mínimas son de $27.5^{\circ} \mathrm{C}$ y $13.7^{\circ} \mathrm{C}$ con una media anual de $21.6^{\circ} \mathrm{C}$.

Materiales de Campo: Se emplearon como referencia las cartas topográficas de escala 1:250,000 y 1: 50,000 (INEGI, 1978) Se utilizó un altímetro para precisar la altitud medida en msnm y un geoposicionador global (GPS), para la ubicación geográfica del mismo, definido en coordenadas UTM (Universal Transverse de Mercator).

Muestreo de la vegetación. Los datos de campo se levantaron basándose en una serie de sitios seleccionados a lo largo de rutas establecidas como brechas, terracerías y carreteras a distancias de 3 a 5 $\mathrm{km}$ entre si, dependiendo de las características de la vegetación presente en el sitio. La metodología consistió en un muestreo sistemático, propuesta por el programa Gap Analysis del Río Grande, planteado por Texas Cooperative Fish and Wildlife Research Unit (TX-CFWRU, 1998). Los sitios de muestreo, fueron en comunidades con un mínimo de 4 ha $y$ relativamente homogéneas.
Tamaño de la muestra. Se evaluaron 110 sitios, cubriendo todos los diferentes tipos de vegetación, en una superficie total de $10780 \mathrm{~km}^{2}$.

Variables evaluadas. Cobertura Vegetal (CV) de las especies vegetales dominante y codominante en el sitio. Se registraron también características fisiográficas y de uso de suelo, que no se contemplan es este estudio.

Determinación de la superficie que ocupan las comunidades vegetales. Para obtener esta información se utilizó una imagen del satélite Landsat 5 TM de siete bandas de información espectral, con fecha del mes de mayo de 1999, a la que se le realizó una clasificación supervisada, con el propósito de establecer límites mínimos y máximos de valores digitales para establecer rangos comunes a las clases vegetales definidos para CV (Góngora y Hernández, 2001). El software utilizado fue el IDRISI 32.

Análisis Estadístico de los Datos. Se utilizó un análisis por conglomerados, el cual permite agrupar la naturaleza geográfica de las especies y de los sitios, usando el \% de la cubierta vegetal, mediante el método de varianza mínima de WARD (Johnson, 2000 \& Zavala, 1986). Los datos de los conglomerados se utilizaron para construir modelos estadísticos a partir de coeficientes de correlación y de regresión lineal (Estrada, 1998).

\section{Resultados y Discusión}

Presencia y frecuencia de especies. Larrea tridentata se encontró en 51 sitios por lo que fue la especie más ampliamente distribuida con una frecuencia de $46.36 \%$, teniendo la mayor asociación con la especie codominante Fouquieria splendens, en 14 sitios, seguida de la especie Prosopis glandulosa, en 13 sitios. Otras asociaciones importantes fueron con Opuntia sp y Parthenium incanum con 7 sitios y Jatropha dioica con 5 sitios (Tabla 1), estos resultados coinciden con lo reportado por Marroquín et al., (1981) que encontraron ampliamente distribuida a $L$. tridentata en las zonas áridas del norte de México.

Otra especie dominante y segunda en importancia fue Acacia constricta que se presentó de manera muy dispersa en 21 sitios del área de estudio con una frecuencia de $19.09 \%$. El mayor grado de asociación se observó con Parthenium incanum, en 8 sitios y con Prosopis glandulosa en 6 sitios. En tercer grado, destacaron como dominantes las especies Prosopis glandulosa con una frecuencia relativa de $12.73 \%$ presente en 14 sitios y Parthenium incanum que ocurrió en 9 sitios y una frecuencia de 8.18\%; generalmente estos estratos se encontraron formando asociaciones con la comunidad de Larrea tridentata principalmente y Acacia sp. (Tabla 1).

Presencia de especies. Se identificaron 27 especies vegetales, 15 dominantes y 21 codominantes en los 110 sitios evaluados. Larrea tridentata, fue la de mayor presencia lo que representa un $63 \%$ de los 
Diciembre 2006

sitios; le sigue Prosopis glandulosa, presente en 37 sitios, equivalente a un $33 \%$ de presencia; otras especies importantes fueron Acacia constricta, Parthenium incanum y Fouquieria splendens, con 28, 24 y 14 apariciones en sitios de muestreo (Tabla 2).

Tabla 1. Presencia y porcentaje de frecuencia de especies vegetales dominantes y codominantes identificadas en los sitios.

\begin{tabular}{|c|c|c|c|c|}
\hline $\begin{array}{c}\text { Especies } \\
\text { dominantes }\end{array}$ & $\begin{array}{l}\text { No. de } \\
\text { sitios }\end{array}$ & $\begin{array}{l}\text { \% de } \\
\text { Frecuencia }\end{array}$ & $\begin{array}{c}\text { Especies } \\
\text { codominantes } \\
\text { asociadas } \\
\end{array}$ & $\begin{array}{l}\text { No. de } \\
\text { sitios }\end{array}$ \\
\hline Larrea tridentata & 51 & 46.36 & $\begin{array}{l}\text { Fouquieria splendens } \\
\text { Prosopis glandulosa } \\
\text { Parthenium incanum } \\
\text { Opuntia sp } \\
\text { Jatropha dioica } \\
\text { Acacias sp } \\
\text { Otras }\end{array}$ & $\begin{array}{c}14 \\
13 \\
7 \\
7 \\
5 \\
3 \\
2\end{array}$ \\
\hline Acacia constricta & 21 & 19.09 & $\begin{array}{l}\text { Parthenium incanum } \\
\text { Prosopis glandulosa } \\
\text { Otras }\end{array}$ & $\begin{array}{l}8 \\
6 \\
7\end{array}$ \\
\hline $\begin{array}{l}\text { Prosopis } \\
\text { glandulosa }\end{array}$ & 14 & 12.73 & $\begin{array}{l}\text { Larrea tridentata } \\
\text { Aloysia gratíssima } \\
\text { Acacia constricta } \\
\text { Otras }\end{array}$ & $\begin{array}{l}6 \\
3 \\
2 \\
3\end{array}$ \\
\hline $\begin{array}{l}\text { Parthenium } \\
\text { incanum }\end{array}$ & 9 & 8.18 & $\begin{array}{l}\text { Larrea tridentata } \\
\text { Acacia constricta } \\
\text { Otras }\end{array}$ & $\begin{array}{l}4 \\
3 \\
2\end{array}$ \\
\hline Otras especies* & 15 & 13.64 & & 95 \\
\hline
\end{tabular}

*Opuntia imbricata, Agave lecheguilla, Yucca carnerosana, Bouteloua gracilis, Hilaria mutica y Aristida sp.

Cobertura vegetal. La especie Larrea tridentata, presentó un promedio de $24.49 \%$ con coberturas muy variables, que desde 5 hasta $60 \%$. Estos valores señalan a esta especie como característica de estas comunidades. Otra especie importante en cobertura fue Acacia constricta con casi $29 \%$ de cobertura vegetal en promedio, esto la convierte junto con Prosopis glandulosa, Parthenium incanum $y$ Fouquieria splendens como las especies de mayor presencia y cubierta vegetal en estas comunidades vegetales.

El resto de las especies encontradas fueron poco frecuentes $y$ aunque algunas presentan coberturas importantes, en estos ecosistemas solo estuvieron presentes muy aisladamente. Esta información permite realizar programas de manejo basados en las especies dominantes y subdominantes, por el nivel de presencia encontrado en este estudio.

Análisis de la CV por Conglomerados. Los resultados muestran un valor de $r^{2}=80.93 \%$ para las coberturas vegetales presentes en los diferentes sitios de observación. Los sitios fueron agrupados en 3 conglomerados a una distancia de $\mathrm{r}^{2}=45.02 \%$, definido por el cluster C3 del grupo 2, al respecto Sierra (2001) señala que los sitios unidos a una menor distancia (nivel 0.0) son más similares entre si, para el caso las pequeñas agrupaciones dentro de los conglomerados, presentan condiciones de $\mathrm{r}^{2}$ $=0.01$, lo que los hace ser de características muy similares.

Se formaron 3 grupos, con diferentes números de sitios. El grupo 1, se compone de 23 sitios a una distancia de $r^{2}$ semiparcial $=0.06$ con dos conglomerados, el C7 y el C17 (Tabla 3).

El C7 presentó 10 sitios, con las siguientes especies: Prosopis glandulosa, Parthenium incanum, Celtis pallida, Hilaria mutica, Agave lecheguilla, Larrea tridentata, Jatropha dioica, Portieria angustifolia, Acacia greggii, Himenoclea monogyra, Opuntia imbricata, Opuntia engelmannii, entre otras, con una $\mathrm{CV}$ de $49.0 \%$ en promedio y rangos de entre $30 \%$ y $70 \%$.

El C17 se constituyó por 13 sitios y por 6 especies: Prosopis glandulosa, Aloysia gratissima, Acacia constricta, Bouteloua gracilis, Larrea tridentata y Acacia greggii. Este conglomerado presentó una $\mathrm{CV}$ promedio de $52.31 \%$ con valores mínimos de $35 \%$ a valores máximos de $75 \%$.

El grupo 2 se integró por una frecuencia de 29 sitios, con un valor de $r^{2}=0.07$, Se determinaron dos conglomerados, el C11con 21 sitios y una $\mathrm{r}^{2}$ semiparcial = 0.016 y el C26 con 8 sitios y una $r^{2}$ semiparcial $=0.005452$.

Las especies que caracterizaron a C11 fueron 10 en el siguiente orden: Acacia constricta, Parthenium incanum, Prosopis glandulosa, Flourensia cernua, Acacia greggii, Larrea tridentata, Aloysia gratissima, Mimosa biuncifera, Hilaria mutica, y Dasylirion leiophyllum; su promedio de cobertura fue de $52.86 \%$. La especie más dominante en este conglomerado es Acacia constricta, y se asoció a otras especies como Prosopis glandulosa y Parthenium incanum, lo que le permitió identificarlo como un conglomerado muy homogéneo.

El conglomerado C26 presentó solo 4 especies, sobresaliendo Parthenium incanum, la cual se encontró como dominante en 8 sitios, asociándose con Acacia constricta y Larrea tridentata principalmente. La CV es de $50.63 \%$ en promedio y un $\mathrm{r}^{2}=0.005452$. 
Tabla 2. Presencia de especies y su cobertura vegetal promedio (\%) en el área de estudio.

\begin{tabular}{|c|c|c|c|}
\hline $\begin{array}{c}\text { No. de } \\
\text { Especies }\end{array}$ & $\begin{array}{c}\text { Especies } \\
\text { identificadas }\end{array}$ & $\begin{array}{c}\text { No. } \\
\text { De } \\
\text { Sitios }\end{array}$ & $\mathrm{CV}$ \\
\hline 1 & Larrea tridentata & 69 & 24.49 \\
\hline 2 & Prosopis glandulosa & 37 & 19.11 \\
\hline 3 & Acacia constricta & 28 & 28.93 \\
\hline 4 & Parthenium incanum & 24 & 25.83 \\
\hline 5 & Fouquieria splendens & 14 & 5.29 \\
\hline 6 & Jatropha dioica & 6 & 15.83 \\
\hline 7 & Opuntia leptocaulis & 5 & 6.00 \\
\hline 8 & Acacia greggii & 5 & 10.00 \\
\hline 9 & Aloysia gratissima & 4 & 26.25 \\
\hline 10 & Opuntia sp & 3 & 10.00 \\
\hline 11 & Yucca carnerosana & 3 & 25.00 \\
\hline 12 & Flourensia cernua & 3 & 31.67 \\
\hline 13 & Hilaria mutica & 3 & 46.67 \\
\hline 14 & Agave lecheguilla & 2 & 25.00 \\
\hline 15 & $\begin{array}{l}\text { Hymenoclea } \\
\text { monogyra }\end{array}$ & 2 & 42.50 \\
\hline 16 & Atriplex canescens & 1 & 2.00 \\
\hline 17 & Mimosa biuncifera & 1 & 5.00 \\
\hline 18 & Porlieria angustifolia & 1 & 5.00 \\
\hline 19 & Acacia neovernicosa & 1 & 10.00 \\
\hline 20 & Celtis pallida & 1 & 15.00 \\
\hline 21 & Opuntia engelmannii & 1 & 15.00 \\
\hline 22 & Bouteloua gracilis & 1 & 20.00 \\
\hline 23 & Opuntia imbricata & 1 & 20.00 \\
\hline 24 & Viguiera stenoloba & 1 & 20.00 \\
\hline 25 & Dasylirion leiophyllum & 1 & 25.00 \\
\hline 26 & Bouteloua-Hilaria & 1 & 35.00 \\
\hline 27 & Bouteloua-Aristida & 1 & 40.00 \\
\hline
\end{tabular}

El grupo 3 se caracterizó por tener 58 sitios siendo el que mayor número presentó, estando ampliamente distribuido en el área de estudio. Presenta un $\mathrm{r}^{2}=$ 0.049 y 13 especies. Se definieron 2 conglomerados, C10 y C6.

El C10 caracterizado por 45 sitios, el de mayor frecuencia dentro del grupo y entre los grupos, el cual mostró una CV de $41.8 \%$, valor intermedio comparado con los demás conglomerados, que lo define como un conglomerado de condición regular en cuanto a CV. Las especies que lo definen son: Larrea tridentata, Prosopis glandulosa, Fouquieria splendens, Parthenium incanum, Opuntia leptocaulis, Jatropha dioica, Opuntia sp, Flourensia cernua y Acacia greggii.

El C6 lo ocuparon 13 sitios, presentó una baja CV con 22.9\%, caracterizándolo las especies Larrea tridentata, Yucca carnerosana, Prosopis glandulosa, Viguiera stenoloba, Opuntia sp, Acacia constricta,
Tabla 3. Grupos determinados por análisis de conglomerados con sus

\begin{tabular}{|c|c|c|c|c|c|}
\hline $\begin{array}{l}\text { No. } \\
\text { Grupo }\end{array}$ & $\begin{array}{l}\text { No. } \\
\text { Sitios }\end{array}$ & $\begin{array}{l}\text { Conglome } \\
\text {-rados }\end{array}$ & $\begin{array}{c}\text { No. especies } \\
\text { dominantes }\end{array}$ & $\begin{array}{l}\text { No. de especies } \\
\text { codominantes }\end{array}$ & $\begin{array}{c}\text { Prome-dio } \\
\text { de CV }\end{array}$ \\
\hline \multirow[b]{2}{*}{1} & 10 & C7 & 7 & 6 & 49.00 \\
\hline & 13 & C17 & 2 & 6 & 52.31 \\
\hline \multirow{2}{*}{2} & 21 & C11 & 1 & 9 & 52.86 \\
\hline & 8 & C26 & 1 & 3 & 50.63 \\
\hline \multirow{2}{*}{3} & 45 & C10 & 2 & 6 & 41.76 \\
\hline & 13 & C6 & 5 & 6 & 22.92 \\
\hline
\end{tabular}

Fouquieria splendens y Atriplex canescens; y se definió como un grupo de mayor disimilaridad dentro y entre grupos.

En términos generales es importante mencionar que los conglomerados explicados y definidos en los tres grupos anteriores, presentaron una primera clasificación vegetal, al respecto Stoms (2001) de GAP analysis, establece que se realice una clasificación jerárquica de $\mathrm{CV}$ de las especies vegetales dominantes, para que estas permitan la interpretación de las clases de vegetación.

En base a una clasificación espectral de la imagen de satélite, se determinaron 12 clases, de las cuales 9 identifican asociaciones o comunidades vegetales, mientras que en el resto no se pueden definir claramente, a lo cual Puga (2003) y Machado (1998) las señalan como categorías desconocidas 0 información alguna.

Dentro de las asociaciones de interés de este estudio sobresalen Larrea con Prosopis con un $24.50 \%$ de la superficie que ocupa el área de estudio, seguida de Prosopis con L tridentata, con un $19.64 \%$ y en tercer lugar se encontró a la asociación $L$. tridentata con Opuntia sp. con un $17.37 \%$. Las superficies que ocupan en el área de estudio se muestra en la Tabla 4.

Un análisis general de la presencia de especies nos indica que en el $67.84 \%$ de la superficie está presente Larrea tridentata, la cual domina en las dos terceras partes de la zona estudiada dentro del ecosistema Desierto Chihuahuense, coincidiendo con Marroquín et al. (1981) que encontraron ampliamente distribuida a esta especie en las zonas áridas del norte de México. 
Tabla 4. Superficie ocupada por diferentes asociaciones vegetales en el área de estudio.

\begin{tabular}{|c|c|c|c|}
\hline $\begin{array}{l}\text { No. de } \\
\text { clases }\end{array}$ & $\begin{array}{l}\text { Asociaciones de especies vegetales } \\
\text { dominantes }\end{array}$ & $\begin{array}{l}\text { Superficie* } \\
\text { ha }\end{array}$ & $\%$ \\
\hline $1-$ & Área sin vegetación aparente & 34006.56 & 3.15 \\
\hline $2-$ & Afloramiento rocoso y Áreas de bosque & 86273.06 & 8.00 \\
\hline 3- & Cultivos y áreas ribereñas & 24422.19 & 2.26 \\
\hline 4- & Larrea tridentata - Opuntia leptocaulis & 59441.69 & 5.51 \\
\hline 5- & Larrea tridentata - Opuntia sp & 187394.13 & 17.37 \\
\hline $6-$ & Acacia constricta - Prosopis glandulosa & 117642.38 & 10.90 \\
\hline 7- & Prosopis glandulosa - Larrea tridentata & 209996.94 & 19.64 \\
\hline 8- & Larrea tridentata - Prosopis glandulosa & 264319.88 & 24.50 \\
\hline 9- & Agave lecheguilla - Larrea tridentata & 8887.50 & 0.82 \\
\hline $10-$ & Hilaria mutica - Prosopis & 15474.13 & 1.43 \\
\hline 11- & $\begin{array}{l}\text { Matorral micrófilo sub inerme } \\
(10 \text { a } 15 \%)\end{array}$ & 44280.81 & 4.10 \\
\hline $12-$ & $\begin{array}{l}\text { Matorral micrófilo sub inerme } \\
\text { (menor al 10\% ) }\end{array}$ & 26920.25 & 2.49 \\
\hline
\end{tabular}

*Superficie del área de estudio:

1079059.50

La segunda especie importante por su presencia es Prosopis glandulosa, que estuvo presente en un $56.72 \%$ de la superficie, abajo solo $10 \%$ de la gobernadora, lo que señala que estas 2 especies dominan este ecosistema.

\section{Conclusiones}

Existe una importante diversidad de especies en este ecosistema desértico ya que se identificaron un total de 27 especies de vegetación dominantes y codominantes.

Las especies dominantes de mayor presencia fueron en orden de importancia: Larrea tridentata, Acacia constricta, Prosopis glandulosa y Parthenium incanum.

Las especies codominantes con mayor presencia fueron: Prosopis glandulosa, Parthenium incanum, Fouquieria splendes y Larrea tridentata.

La cobertura vegetal en orden de importancia, la presentaron las especies: Acacia constricta 28.93\%, Parthenium incanum $25.83 \%$ Larrea tridentata $24.49 \%$ y Prosopis glandulosa con $19.11 \%$.

El análisis por conglomerados de los sitios, permitió agrupar de manera eficiente a los sitios encontrados, formando grupos homogéneos y conglomerados que facilitan un análisis de la estructura de estas comunidades, lo cual permitirá un adecuado manejo para garantizar la sustentabilidad de estas comunidades vegetales.

Las especies con mayor presencia en este ecosistema son: Larrea tridentata y Prosopis glandulosa, localizadas en más de la mitad de la superficie del área de estudio.

\section{Literatura Citada}

Chilar J., Latifovic R., Beaubien J., Li Z. \& Magnussen S. 2000. Selecting representative high resolution simple image for land cover studies. Part 2: Application to estimating land cover composition. Remote sensing of environment. 72: 127-138.

Comisión Nacional de Agua, \& SEMARNAP. 2000. El agua en México: Retos y avances. México, D. F.

Estrada C.A.E. 1995. Flora de la cuenca de la laguna de Babícora, Municipios de Gómez Farias y Madera, Chihuahua. Tesis Maestría. Facultad de Zootecnia. Univ. Autónoma de Chihuahua. Chihuahua, Chih. México.

Estrada C.A.E. 1998. Ecología del matorral submontano en el estado de Nuevo León. Disertación Doctoral. Facultad de Zootecnia. Univ. Autónoma de Chihuahua. Chihuahua, Chih. México.

Fierro G.L.C., Ibarra F., Martín M.H., Melgoza A., Peña J.M., Sierra J.S. \& Soltero S. 1980. Manual de métodos de muestreo de vegetación. Instituto Nacional de Investigaciones Pecuarias-S. A. R. H. México.

Góngora J.J. \& Hernández R. 2001. Estadística Descriptiva. Ed. Trillas. México, D. F.

González P.A. 2001. Caracterización ecológica de la vegetación en las áreas de protección de flora y fauna Cañón de Santa Elena y Big Bend Nacional Park. Disertación Doctoral. Facultad de Zootecnia. Univ. Autónoma de Chihuahua. Chihuahua, Chih. México.

INEGI. 1976. Carta Topográfica. Escala 1:50 000. $1^{\text {a }}$ ed. H13D32, H13D42, H13D43, H13D52, H13D53, H13D62, H13D63, H13D64. CETENAL. Aguas Calientes, México.

INEGI. 1977. Carta Topográfica. Escala 1:50 000. $1^{\text {a }}$ ed. H13D54. CETENAL. Aguas Calientes, México.

INEGI 1978. Carta Topográfica. Escala 1:50 000. $1^{\text {a }}$ ed. H13D33, H13D34, H13D44. DETENAL. México, D. F.

Johnson D.E. 2000. Métodos Multivariados Aplicados al Análisis de Datos. Editores International Thomson. México.

Krebs C.J. 1985. Ecología - Estudio de la Distribución y la Abundancia. 2a ed. Editorial Harla. México.

Krebs C.J. 1998. Ecological Methodology. $2^{\text {a }}$ ed. Publicaciones Addison-Welsey. Menlo Park, Canadá.

Machado G.M. 1998. Análisis de la vegetación en el Cañón de San Matías, Baja California a través de una Imagen Landsat-TM. Tesis Maestría. Instituto de Ciencias Agrícolas. Univ. Autónoma de Baja California. Mexicali, Baja California. México.

Marroquín S.J., Borja G.L., Velásquez R.C. \& de la Cruz J.A. 1981. Estudio Ecológico Dasonómico de las Zonas Áridas del Norte de México. Publicación especial No. 2. 2a ed.

Ondarza R.N. 1993. Ecología: El Hombre y su Ambiente. Editorial Trillas. México.

Puga S. 2003. Caracterización de la vegetación e identificación de especies arbóreas dominantes en la región de la alta Babícora utilizando imágenes de satélite Ikonos. Tesis Maestría. Facultad de Zootecnia. Univ. Autónoma de Chihuahua. Chihuahua, Chih. México.

Rzedowski J. 1978. Vegetación de México. Editorial LIMUSA. México. 
Sierra T.J.S. 2001. Análisis ecológico de las comunidades de saladillo (Atriplex acanthocarpa) en el Estado de Chihuahua y Suroeste de Coahuila. Disertación Doctoral. Facultad de Zootecnia. Univ. Autónoma de Chihuahua. Chihuahua, Chih. México.

Solís E.J.A. 1992. Inventario de vegetación en el Ejido Charcos de risa Municipio de Francisco I. Madero, Coahuila. Tesis de Licenciatura. Escuela de Ciencias Biológicas. Univ. Autónoma de Coahuila. Torreón, Coah. Méx.

Soto C.R.A., Castañeda C., Garibay L. \& Parra A. 2000. Manual de Educación Ambiental para Comunidades del Desierto Chihuahuense. Facultad de Zootecnia. Univ. Autónoma de Chihuahua. Chihuahua, Chih. México.
Stoms D. 2001. Estrato de información de la vegetación actual. Manual de Gap Analysis. Ed. Español. USGS. Santa Bárbara, CA. U.S.A.

TX-CFWRU. 1998. Gap analysis Río Grande: Estrategia general para el muestreo de la vegetación en la región fronteriza del Río Bravo. Univ. Texas Tech. Lubbock, Tx. U.S.A.

Whittaker R.H. 1972. Communities and Ecosystems. 5a impresión. McMillan Company. New York. U.S.A.

Zavala H.J.A. 1986. Introducción al enfoque multivariado en estudios de vegetación. Instituto Nacional de Investigaciones sobre Recursos Bióticos. Xalapa, Ver. Méx.

${ }^{1}$ Universidad Autónoma de Chihuahua, Facultad de Zootecnia, msosac@uach.mx

Periférico Fco R. Almada Km. 1.Teléfono (614) 4340303 Fax 4340345

${ }^{2}$ Estudiante graduado. Universidad Autónoma de Chihuahua.

Periférico Fco R. Almada Km. 1.Teléfono (614) 4340303 Fax 4 340345, jgala55@yahoo.com.mx

${ }^{3}$ Universidad Autónoma de Chihuahua, Facultad de Zootecnia, Periférico Fco R. Almada Km. 1.Teléfono (614) 4340303 Fax 4 340345, tlebgue@uach.mx

${ }^{4}$ Universidad Autónoma de Chihuahua, Facultad de Zootecnia, Periférico Fco R. Almada Km. 1.Teléfono (614) 4340303 Fax 4340345 rsoto@uach.mx

${ }^{5}$ Universidad Autónoma de Chihuahua, Facultad de Zootecnia, Periférico Fco R. Almada Km. 1.Teléfono (614) 4340303 Fax 4340345 spuga2002@yahoo.com 